\title{
A Possible Approach to Inclusion of Space and Time in Frame Fields of Quantum Representations of Real and Complex Numbers
}

\author{
Paul Benioff \\ Physics Division, Argonne National Laboratory, Argonne, IL 60439, USA \\ Correspondence should be addressed to Paul Benioff, pbenioff@anl.gov \\ Received 15 May 2009; Revised 26 August 2009; Accepted 27 August 2009 \\ Recommended by Shao-Ming Fei \\ This work is based on the field of reference frames based on quantum representations of real and \\ complex numbers described in other work. Here frame domains are expanded to include space \\ and time lattices. Strings of qukits are described as hybrid systems as they are both mathematical \\ and physical systems. As mathematical systems they represent numbers. As physical systems in \\ each frame the strings have a discrete Schrodinger dynamics on the lattices. The frame field has \\ an iterative structure such that the contents of a stage $j$ frame have images in a stage $j-1$ (parent) \\ frame. A discussion of parent frame images includes the proposal that points of stage $j$ frame \\ lattices have images as hybrid systems in parent frames. The resulting association of energy with \\ images of lattice point locations, as hybrid systems states, is discussed. Representations and images \\ of other physical systems in the different frames are also described.
}

Copyright (C) 2009 Paul Benioff. This is an open access article distributed under the Creative Commons Attribution License, which permits unrestricted use, distribution, and reproduction in any medium, provided the original work is properly cited.

\section{Introduction}

The need for a coherent theory of physics and mathematics together arises from considerations of the basic relationship between physics and mathematics. Why is mathematics relevant to physics [1-3]? One way to see the problem is based on the widely held Platonic view of mathematics. If mathematical systems have an abstract ideal existence outside of space and time and physics describes the property of systems in space and time, then why should the two be related at all? Yet it is clear that they are very closely related.

The problem of the relationship between the foundations of mathematics and physics is not new. Some recent work on the subject is described in [4-6] and in [7-9]. In particular the work of Tegmark [8] is is quite explicit in that it suggests that the physical universe is a mathematical universe. 
Another approach to this problem is to work towards construction of a theory that treats physics and mathematics together as one coherent whole [10,11]. Such a theory would be expected to show why mathematics is so important to physics by describing details of the relation between mathematical and physical systems.

In this paper a possible approach to a coherent theory of physics and mathematics is described. The approach is based on the field of reference frames that follows from the properties of quantum mechanical representations of real and complex numbers $[12,13]$.

The use, here, of reference frames is similar in many ways to that used by different workers in areas of physics [14-19]. In general, a reference frame provides a background or basis for descriptions of systems. In special relativity, reference frames for describing physical dynamics are based on choices of inertial coordinate systems. In quantum cryptography, polarization directions are used to define reference frames for sending and receiving messages encoded in qubit string states.

The use of reference frames here differs from those noted above in that the frames are not based on a preexisting space and time as a background. Instead they are based on a mathematical parameterization of quantum theory representations of real and complex numbers. In particular, each frame $F_{j, k, g}$ in the field is based on a quantum theory representation, $R_{j, k, g}, C_{j, k, g}$ of the real and complex numbers where $R_{j, k, g}$ can be viewed as a set of equivalence classes of Cauchy sequences of quantum states of qukit strings. $C_{j, k, g}$ is a set of pairs of these equivalence classes. The parameter $k \geq 2$ is the base ( $k=2$ for qubits), $g$ denotes a basis choice for the states of qukit strings that are values of rational numbers, and $j$ denotes an iteration stage. The existence of iterations follows from the observation that the representations of real and complex numbers are based on qukit string states. These are elements of a Hilbert space that is itself defined as a vector space over a field of complex numbers. Consequently one can use the real and complex numbers constructed in a stage $j$ frame as the base of a stage $j+1$ frame.

Each reference frame contains a considerable number of mathematical structures. Besides $R_{j, k, g}$ and $C_{j, k, g}$, a frame $F_{j, k, g}$ contains representations of all mathematical systems that can be described as structures based on $R_{j, k, g}$ and $C_{j, k, g}$. However frames do not contain physical theories as mathematical structures based on $R_{j, k, g}, C_{j, k, g}$. The reason is that the frames do not contain any representations of space and time.

The goal of this paper is to take a first step in remedying this defect by expansion of the domain of each frame to include discrete space and time lattices. The lattices, $L_{j, k, L, m}$, in a frame, $F_{j, k, g}$, are such that the number of points in each dimension is given by $k^{L}$, the spacing $\Delta=k^{-m}$ and $m=0,1, \ldots, L$. For each lattice, $L$ and $m$ are fixed with $L$ being an arbitrary nonnegative integer. It follows that each dimension component of the location of each point in a lattice is a rational number expressible as a finite string of base $k$ digits.

Representations of physical systems of different types are also present in each frame. However, the emphasis here is on strings of qukits, $S_{j, k^{\prime}, L^{\prime}, m^{\prime}}$, present in each frame. These strings are considered to be hybrid systems in that they are both physical systems and mathematical systems. As mathematical systems, the quantum states of each string, in some basis, represent a set of rational numbers. As physical systems the motion of strings in a frame is described relative to a space and time lattice in the frame. This dual role is somewhat similar to the concept that information is physical [20].

Considerable space in the paper is devoted to how observers in a stage $j-1$ (parent) frame view the contents of a stage $j$ frame. For an observer, $O_{j}$, in a frame $F_{j, k, g}$ the numbers in the real and complex number base of the frame are abstract and have no structure. The only requirement is that they satisfy the set of axioms for real or complex number systems. 
Points of lattices $L_{j, k, L, m}$ in the frame are also regarded as abstract and without structure. The only requirement is that the lattices satisfy some relevant geometric axioms.

The view of the contents of a stage $j$ frame as seen by an observer, $O_{j-1}$, in a stage $j-1$ frame, $F_{j-1, k^{\prime}, g^{\prime}}$, is quite different. Elements of the stage $j$ frame that $O_{j}$ sees as abstract and with no structure are seen by $O_{j-1}$ to have structure. Numbers in $R_{j, k, g}$ are seen by $O_{j-1}$ to be equivalence classes of Cauchy sequences of states of stage $j-1$ hybrid systems. Space points of stage $j$ lattices with $D$ space and one time dimension are seen in a stage $j-1$ frame to be $D$ tuples of hybrid systems with the location of each point given by a state of the $D$ tuple. Time points are seen to be hybrid systems whose states correspond to the possible lattice time values.

All this and more is discussed in the rest of the paper. The next section is a brief summary of quantum theory representations and the resulting frame fields $[12,13]$. Section 3 describes a possible approach to a coherent theory of physics and mathematics as the inclusion of space and time lattices in each frame of the frame field. Properties of the lattices in the frames are described. Qukit strings as hybrid systems are discussed in the next section. Their mathematical properties as rational number systems with states as values of rational numbers are described. Also included is a general Hamiltonian description of the rational number states as energy eigenvalues and a Schrödinger equation description of the dynamics of these systems.

Section 5 describes frame entities as viewed from a parent frame. Included is a description of real and complex numbers, quantum states and Hilbert spaces, and space and time lattices. Section 6 discusses in more detail a stage $j-1$ views of stage $j$ lattice points and locations as tuples of hybrid systems and point locations as states of the tuples. Dynamics of these tuples in stage $j-1$ is briefly described as is the parent frame view of the dynamics of physical systems in general.

The last section is a discussion of several points. The most important one is that frame field description given here leads to a field of different descriptions of the physical universe, one for each frame, whereas there is just one. This leads to the need to find some way to merge or collapse the frame field to correspond to the accepted view of the physical universe. This is discussed in the section as are some other points.

Whatever one thinks of the ideas and systems described in this work, it is good to keep the following points in mind. One point is that the existence of the reference frame field is based on properties of states of qukit string systems representing values of rational numbers. However the presence of a frame field is more general in that it is not limited to states of qukit strings. Reference frame fields arise for any quantum representation of rational numbers where the values of the rational numbers, as states of some system, are elements of a vector space over the field of complex numbers.

Another point is that the three-dimensional reference frame field described here exists only for quantum theory representations of the natural numbers, the integers, and the rational numbers. Neither the basis degree of freedom $g$ nor the iteration stages, $j$, are present in classical representations. This is the case even for classical representations based on base $k$ digit or kit strings. The reason is that states of digit strings are not elements of a vector space over a complex number field.

Finally, although understandable, it is somewhat of a mystery why so much effort in physics has gone into the description of various aspects of quantum geometry and space time and so little into quantum representations of numbers. This is especially the case when one considers that natural numbers integers, rational numbers, and probably real and complex numbers are even more fundamental to physics than is geometry. 


\section{Review: Quantum Theory Representations of Numbers and Frame Fields}

\subsection{Quantum Theory Representations of Numbers}

\subsubsection{Representations of Natural Numbers, Integers, and Rational Numbers}

In earlier work $[12,13]$ quantum theory representations of natural numbers $(N)$, integers $(I)$, and rational numbers $(R a)$ were described by states of finite length qukit strings that include one qubit. To keep the description as simple as possible, the strings are considered to be finite sets of qukits and one qubit with the qukits and qubit parameterized by integer labels. The natural ordering of the integers serves to order the set into a string.

This purely mathematical representation of qukit strings makes no use of physical representation of qukit strings as extended systems in space and/or time. Physical representations are described later on in Section 4 after the introduction of space and time lattices into each frame of the frame field.

The qukit $\left(q_{k}\right)$ string states are given by $|\gamma, s\rangle_{k, L, m, g}$ where $s$ is a $0,1, \ldots, k$-1-valued function with domain $0, \ldots, L-1$, and $\gamma=+,-$ denotes the sign. The string location of the sign qubit is given by $m$ where $m=0, \ldots, L$. $L$ is any nonnegative integer. This expresses the range of possible locations of the sign qubit from one end of the string to the other. By convention $m=0$ has the sign qubit at the right end of the string, $m=L$ at the left end. The qubit can occupy the same integer location as a qukit. The reason for the subscript $g$ will be clarified later on.

A compact notation is used where the location $m$ of the sign qubit is also the location of the $k$-al point. As examples, the base 10 numbers $3720,-.0474,-12.71$ are represented here by $|3720+\rangle,|-0474\rangle$, and $|12-71\rangle$, respectively.

Strings are characterized by the values of $k, L, m$. For each $k, L$, and $m$ the string states $|\gamma, s\rangle_{k, L, m, g}$ give a unified quantum theory representation of natural numbers and integers in $N_{k, L}, I_{k, L}$, and $R a_{k, L, m}$. For numbers in $N_{k . L}, \gamma=+, m=0$; for numbers in $I_{k, L}, m=0$, and there are no restrictions for $R a_{k, L, m}$. Here $R a_{k, L, m}$ is the set of rational numbers expressible as $l \Delta$ where $l$ is any integer whose absolute value is $<k^{L}$ and $\Delta=k^{-m} . I_{k, L}=R a_{k, L, 0}$ and $N_{k, L}$ is the set of nonnegative integers in $I_{k, L}$. The correspondence between the numbers $l \Delta$ and the states $|\gamma, s\rangle_{k, L, m, g}$ is given by the observation that each $s$ corresponds to an integer $l=\sum_{j=0}^{L-1} s(j) k^{j}$. Also, as noted, $m$ is the location of the $k-a l$ point measured from the right end of the string.

Since one is dealing with quantum states of qukit strings, states with leading and trailing $0 s$ are included. In this case there are many states that are all arithmetically equal even though they are orthogonal quantum mechanically. For example, $|013-470\rangle={ }_{A}|13-47\rangle$ even though the two states are orthogonal.

The set of states so defined form a basis set that spans a Fock space $\mathcal{F}_{k}$ of states. A Fock space is used because the basis set includes states of $q_{k}$ strings of different lengths. (Representations of these states by use of qukit annihilation creation operators will not be done here as it is not needed for this paper. Representations in terms of these operators for different types of numbers are described in $[12,21]$. Also see [22].) Linear superposition states in the space have the form

$$
\psi=\sum_{L, m} \sum_{\gamma, s} c_{\gamma, s, L, m}|\gamma, s\rangle_{k, L, m, g}
$$


The $L$ and $m$ sums are over all positive integers and from 0 to $L$, and the $s$ sum is over all functions with domain $[0, L-1]$.

As already noted, the states $|\gamma, s\rangle_{k, L, m, g}$ of $q_{k}$ strings are values of rational numbers. Quantum mechanically they also represent a basis choice of states in the Fock space $\mathcal{F}_{k}$. However the choice of a basis is arbitrary in that there are an infinite number of possible choices. Here the choice of a basis is parameterized by the variable $g$. Since choice of a basis is equivalent to fixing a gauge, $g$ is also a gauge fixing parameter.

One can also describe gauge and base transformation operators on these states. Gauge transformations correspond to a basis change $\left(g\right.$ to $\left.g^{\prime}\right)$ and base transformations correspond to a base change $\left(k\right.$ to $\left.k^{\prime}\right)$. Details are given in [13].

\subsubsection{Real and Complex Numbers}

Quantum theory representations of real numbers are defined here as equivalence classes of Cauchy sequences of states of finite $q_{k}$ strings that are values of rational numbers. Let $\psi$ be a function on the natural numbers such that for each $n \psi(n)$ is a basis state:

$$
\psi(n)=\left|\gamma_{n}, s_{n}\right\rangle_{k, L_{n}, m_{n}, g}
$$

For each $n$ the interval $\left[0, L_{n}-1\right]$ is the domain of $s_{n}$.

The sequence $\psi$ is a Cauchy sequence if it satisfies the Cauchy condition.

For each $\ell$ there is a $p$ where for all $j, h>p$,

$$
\left|\left(\left|\psi(j)-{ }_{A, k, g} \psi(h)\right|_{A, k, g}\right)\right\rangle_{k, g}<_{A, k, g}|+,-\ell\rangle_{k, g} .
$$

Here $\left|\left(\left|\psi(j)-{ }_{A, k, g} \psi(h)\right|_{A, k, g}\right)\right\rangle_{k, g}$ is the basis state that is the base $k$ arithmetic absolute value of the state resulting from the arithmetic subtraction of $\psi(h)$ from $\psi(j)$. The Cauchy condition says that this state is arithmetically less than or equal to the state $|+,-\ell\rangle_{k, g}=$ $\left|+, 0_{[0,-\ell+1]} 1_{-\ell}\right\rangle_{k, g}$ for all $j, h$ greater than some $p$. Here $|+,-\ell\rangle$ is a string state that represents the number $k^{-\ell}$. The subscripts $A, k, g$ in the definition of the Cauchy condition indicate that the operations are arithmetic and are defined for base $k$ string states in the gauge $g$. They are not the usual quantum theory operations.

The definition can be extended to sequences $\psi$ of linear superpositions of basis states. In this case one defines a probability $P_{j, h, \ell}$ as a sum over all components of $\psi(j)$ and $\psi(h)$ that satisfy the second line of (2.3). The state $\psi$ is Cauchy if the probability $P_{\psi}=1$ where

$$
P_{\psi}=\liminf _{\ell \rightarrow \infty} \limsup _{p \rightarrow \infty} \inf _{j, h>p} P_{j, h, \ell}
$$

Two sequences $\psi$ and $\psi^{\prime}$ are equivalent, $\psi \equiv \psi^{\prime}$, if the sequence defined by the termwise arithmetic difference of $\psi$ and $\psi^{\prime}$ converges to 0 . The specific condition for this is expressed by (2.3) if one replaces $\psi(h)$ with $\psi^{\prime}(h)$. The relation $\psi \equiv \psi^{\prime}$ is used to define equivalence classes of Cauchy sequences of $q_{k}$ string states. The set of these equivalence classes is a quantum theory representation $R_{k, g}$ of the real numbers. 
Quantum equivalence classes of Cauchy sequences of states are larger than classical equivalence classes because each quantum equivalence class contains many sequences of states that have no classical correspondent. However no new equivalence classes appear. This is a consequence of the fact that each quantum equivalence class contains a basis-valued sequence that corresponds to a classical sequence of finite base $k$ digit strings.

One can also define a canonical representation of each equivalence class as a sequence $\psi(n)$ of basis states such that if $m>n$, then $\psi(n)$ is an initial part of $\psi(m)$. This is similar to the usual classical canonical representation of an equivalence class of real numbers as an infinite string of digits with a $k-a l$ point. The quantum representation would be an infinite string of qukits with each qukit in one of $k$ basis states.

Extension of the above to include quantum representations of complex numbers is straightforward. One method represents complex rational numbers by pairs of states of finite $q_{k}$ strings. This what is actually done in computations involving complex numbers. The state components of the pair represent values of real and imaginary rational numbers.

Cauchy sequences of these state pairs are defined by applying the Cauchy condition separately to the component sequences of real rational number states and imaginary rational number states. Two Cauchy sequences $\psi$ and $\psi^{\prime}$ of state pairs are equivalent, $\psi \equiv \psi^{\prime}$, if the termwise arithmetic differences of the real parts, $\operatorname{Re} \psi{ }_{A} \operatorname{Re} \psi^{\prime}$, and of the imaginary parts, $\operatorname{Im} \psi{ }_{A} \operatorname{Im} \psi^{\prime}$ each converge to 0 . The resulting set of equivalence classes of Cauchy sequences is a quantum theory representation of the set of complex numbers.

\subsection{Fields of Iterated Reference Frames}

Quantum theory representations of real and complex numbers differ from classical representations in two important ways. One is the presence of the gauge freedom or basis choice freedom. This is indicated by the $g$ subscript in $R_{k, g}, C_{k, g}$.

The other difference is based on the observation that states of qukit strings are elements of a Hilbert space or a Fock space. From a mathematical point of view these spaces are vector spaces over the fields of real and complex numbers. It follows from what has been shown that $q_{k}$ string states, as elements of a vector space over the field of real and complex numbers, can be used to construct other representations of real and complex numbers. This suggests the possibility of iteration of the construction described here as the quantum representations of real and complex numbers can in turn be used as the base of Hilbert and Fock spaces for a repeated construction. Here the iteration stage is another degree of freedom for the frame field.

The third degree of freedom arises from the free choice of the base choice for the humber representation. This choice, denoted by the $k$ subscript, is common to both quantum and classical representations.

These three degrees of freedom can be combined to describe a three-dimensional reference frame field. Each reference frame $F_{j, k, g}$ is based on a quantum representation $R_{j, k, g}, C_{j, k, g}$ of the real and complex numbers. The subscripts denote the iteration stage $j$, the base $k$, and the gauge $g$. Each reference frame contains representations of Hilbert and Fock spaces as mathematical structures over $R_{j, k, g}, C_{j, k, g}$.

Because the iteration degree of freedom is directed, it is useful to use genealogical terms to describe the iteration stages. Frames that are ancestors to a given frame $F_{j, k, g}$ occur at stages $j^{\prime}$ where $j^{\prime}<j$. Frames that are descendants occur at stages $j^{\prime}$ where $j^{\prime}>j$. 
From a mathematical point of view there are several possibilities for the stages. The frame fields can have a finite number of stages with both a common ancestor frame and a set of terminal frames. The fields can also be one way infinite either with a common ancestor and no terminal stage or with a terminal stage and no common ancestor, or they can be two way infinite. They can also be cyclic. These last two cases have no common ancestor or terminal frames.

Another way to illustrate the frame field structure is to show, schematically, frames emanating from frames. Figure 1 illustrates a slice of the frame field for a fixed value of $j$. Each point $k^{\prime}, g^{\prime}$ in the $k-g$ plane denotes a reference frame $F_{j+1, k^{\prime}, g^{\prime}}$ at the next iteration stage with $R_{j+1, k^{\prime}, g^{\prime}}, C_{j+1, k^{\prime}, g^{\prime}}$ as its real and complex number base.

Three different viewpoints of the real and complex numbers as frame bases are of use here. $R_{j, k, g}$ and $C_{j, k, g}$ are a view from outside the frame field that denotes the position of the numbers with respect to the field degrees of freedom. To an observer inside $F_{j, k, g}$ the elements of $R_{j, k, g}, C_{j, k, g}$ are seen as external, abstract, featureless elements. They have no structure other than that which follows from the requirement that they satisfy the axioms for real and complex numbers. This assumption is made because it is the view held, at least implicitly by physicists. It also corresponds to how numbers are treated in physical theories. The only properties of numbers relevant to theories are those derived from the appropriate axioms.

An observer in a parent frame $F_{j-1, k^{\prime}, g^{\prime}}$ sees the elements of $R_{j, k, g}, C_{j, k, g}$ as having structure. They are seen as equivalence classes of Cauchy sequences of states of finite $q_{k}$ strings. This is in addition to their having properties derived from the relevant axiom sets.

\section{A Possible Approach to a Coherent Theory of Physics and Mathematics}

The main consideration of this paper is the proposed use of the reference frame field as a possible approach to a coherent theory of physics and mathematics. This ensures that quantum theory representations of natural numbers, integers, rational numbers, and real and complex numbers will play a basic role in the theory.

So far the reference frames contain mathematical systems. These include quantum theory representations of numbers, qukit strings, and representations of other mathematical systems as structures based on the different types of numbers. Physical theories and systems are not present in the frames. The reason is that there are no representations of space and time in the frames. These are needed for theories to describe the kinematics and dynamics of systems moving in space and time.

This must be remedied if the frame field is to be an approach to a coherent theory. One way to fix this is to expand the domains of the frames to include physical systems and descriptions of their dynamics.

\subsection{Space and Time Lattices in Reference Frames}

A first step in this direction is to expand the domain of each frame in the field to include discrete lattices of space and time. The reason for working with discrete instead of continuum space and time will be noted later.

To be more specific, each frame $F_{j, k, g}$ includes a set $\mathcal{L}_{j, k}$ of space and time lattices. Each lattice $L_{j, k, M, \Delta}$ in the set is such that the number $M$ of points in each dimension is finite and 


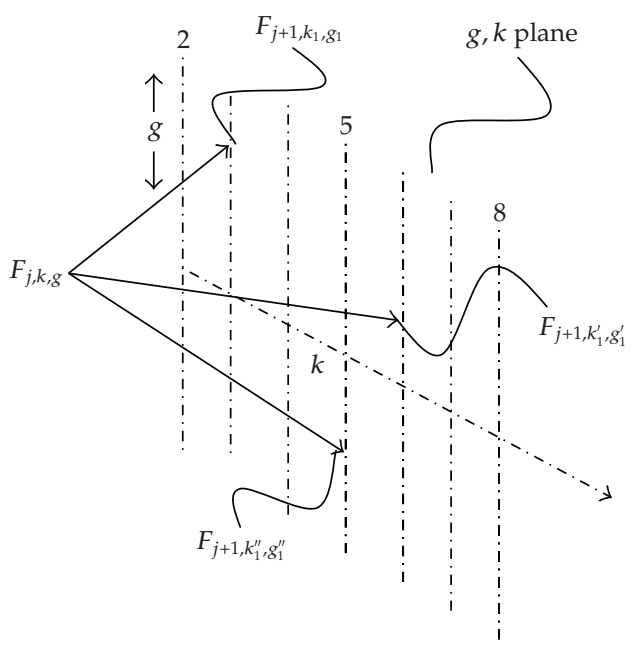

Figure 1: Schematic illustration of frames coming from frame $F_{j, k, g}$. Each of the stage $j+1$ frames is based on quantum representations of real and complex numbers as equivalence classis of Cauchy sequences of qukit string states in $F_{j, k, g}$. The distinct vertical lines in the $k, g$ plane denote the discreteness of the integral values of $k \geq 2$. Only three of the infinitely many frames coming from $F_{j, k, g}$ are shown. Here $k$ denotes the qukit base and $g$ denotes a gauge or basis choice.

the spacing $\Delta$ of points is also finite. In this paper the number $D$ of space dimensions in the lattice will be arbitrary. To keep things simple, the number $M$ of points and spacing $\Delta$ in each of the $D$ space dimensions and the time dimension will be assumed to be the same.

It should be noted that, to an observer in a frame, the points in each space and time lattice in a frame are (emphasis on "are") points of space and time relative to that frame. They are not merely mathematical representations or descriptions of some external space and time. In addition an observer in a frame sees the points of space and time lattices in the frame as abstract, featureless points with no structure. The only requirement is that the lattices should satisfy appropriate geometrical axioms.

A restriction on the lattices in frames is that the values of $M$ and $\Delta$ for each space and time lattice in the set $\mathcal{L}_{j, k}$ are given by

$$
\begin{aligned}
& M=k^{L}, \\
& \Delta=k^{-m} .
\end{aligned}
$$

Here $L$ is an arbitrary nonnegative integer, $m=0,1, \ldots, L$, and $k$ is the same integer $\geq 2$, that is, the $k$ subscript in the frame label, as in $F_{j, k, g}$. Because of this restriction the lattices $L_{j, k, M, \Delta}$ will be labeled from now on as $L_{j, k, L, m}$. Based on (3.1), the location of each space or time point in each dimension $z$ is given by a rational number value, $x_{z}=l_{z} \Delta$, where $l_{z}$ is a nonnegative integer $<M$.

Even though these requirements might seem restrictive, they are sufficiently general to allow lattices of arbitrarily small spacing and arbitrarily many points. Also they can be used to describe sequences of lattices that become continuous in the limit. An example of such a sequence is given by setting $m=[L / 2]$ and increasing $L$ without bound. 
It follows from this description that the points of a lattice, $L_{j, k, L, m}$, with $D$ space and one time dimension can be taken to be $D+1$-tuples of rational numbers. The value of each number in the tuple is expressible as a finite string of base $k$ digits.(Here, unlike the usual case, the frames do not include a continuum space and time as a common background for the lattices in $\mathcal{L}_{j, k}$. One may hope that the structure of space and time, as a continuum or as some other structure, will emerge when one finds a way to merge the frames in the frame field.)

So far each frame contains in its domain, space and time lattices, and strings of qukits that are numbers. It is reasonable to expect that it also contains various types of physical systems. For the purposes of this paper the types of included physical systems do not play an important role as the main emphasis here is on qukit string systems. Also in this first paper descriptions of system dynamics will be limited to nonrelativistic dynamics.

It follows from this that each frame includes a description of the dynamics of physical systems based on the space and time lattices in the frame. The kinematics and dynamics of the systems are expressed by theories that are present in the frame as mathematical structures over the real and complex number base of the frame. This is the case irrespective of whether the physical systems are particles, fields, or strings or have any other form.

One reason that space and time are described as discrete lattices instead of as continua is that it is not clear what the appropriate limit of the discrete description is. As is well known, there are many different descriptions of space and time that are present in literature. The majority of these descriptions arise from the need to combine quantum mechanics with general relativity. They include use of various quantum geometries [23-27] and space time as a foam [28-31] and as a spin network as in loop quantum gravity [32]. These are in addition to the often used assumption of a fixed flat space and time continuum that serves as a background arena for the dynamics of all physical systems, from cosmological to microscopic. Space and time may also be emergent in an asymptotic sense [33].

The fact that there are many different lattices in a frame each characterized by different values of $L$ and $m$ and that each can serve as a background space and time is not a problem. There is no different than the fact that one can use many different space and time lattices with different spacings and numbers of points to describe discrete dynamics of systems.

\section{Qukit String Systems as Hybrid Systems}

So far the domain of each frame contains space and time lattices, many types of physical systems (such as electrons, nuclei, and atoms) and physical theories as mathematical structures based on the real and complex numbers. These describe the kinematics and dynamics of these systems on the lattices. Also included are qukit strings. States of these strings were seen to be values of rational numbers. These were used to describe real and complex numbers as Cauchy sequences of these states.

Here it is proposed to consider these strings as systems that can either be numbers, that is, mathematical systems, or be physical systems. Because of this dual role, they are referred to as hybrid systems. As such they will be seen to play an important role.

Support for this proposal is based on the observation that the description of qukit strings as both numbers and physical systems is not much different than the usual view in physics regarding qubits and strings of qubits. As a unit of quantum information, the states of a qubit can be $|0\rangle$ and $|1\rangle$ which denote single digit binary numbers. The states can also be $|\uparrow\rangle,|\downarrow\rangle$ as spin projection states of a physical spin $1 / 2$ system. In the same way strings of qubits are binary numbers in quantum computation, or they can represent physical systems 
such as spins or atoms in a linear ion trap [34]. To be blunt about it, "Information is Physical" [20], and information is mathematical.

Also it is reasonable to expect that the domain of a coherent theory of physics and mathematics together would contain systems that are both mathematical systems and physical systems. The hybrid systems are an example of this in that they are number systems, which are mathematical systems, and they are physical systems.

Let $S_{j, k^{\prime}, L, m}$ denote a hybrid system in $F_{j, k, g}$ that contains $L$ qukits and one qubit. $L$ is any nonnegative integer, $m$ is any nonnegative integer $\leq L, j$ is the iteration stage of any frame containing these systems, and $k^{\prime}$ is the base (or dimension of the Hilbert state space) of the $q_{k^{\prime}}$ in the string system. Note that $k^{\prime}$ can be different from the base $k$ of the frame $F_{j, k, g}$ containing these systems.

The different $q_{k^{\prime}}$ in the system are distinguished by labels in the integer interval $[1, L]$. The qubit has label $m$ where $0 \leq m \leq L$. The canonical ordering of the integers serves to order the $q_{k^{\prime}}$ and qubit into a string system.

The presence of the sign qubit is needed if the states of the hybrid systems are to be values of rational numbers. Since the qubit also corresponds to the $k^{\prime}-a l$ point, the value of $m$ gives the location of the sign and $k^{\prime}-a l$ point in $S_{j, k^{\prime}, L, m}$.

Note that there is a change of emphasis from the usual description of numbers. In the usual description, strings of base $k^{\prime}$ digits, such as 1423.45 with $k^{\prime}=10$, are called rational numbers. Here states, such as $|1423+45\rangle_{k, g}$, are called values of rational numbers. The hybrid system will also be referred to as a rational number system. The reason is that the set of all basis states of a hybrid system correspond to a set of $k^{L}$ values of rational numbers. The type of number $N, I, R a$, represented is characterized by the value of $m$ and state of the sign qubit in $S_{j, k^{\prime}, L, m}$. (One would like to call $S_{j, k^{\prime}, L, m}$ a rational number instead of a rational number system. This would agree with the usual physical description of systems. For example, a physical system of a certain type is a proton, not a proton system. However referring to $S_{j, k^{\prime}, L, m}$ as a rational number instead of a rational number system seems so odds with the usual use of the term that it is not done here.)

As was noted, the states of the $S_{j, k^{\prime}, L, m}$ systems in a frame $F_{j, k, g}$ are elements of a Hilbert space $\mathscr{\ell}_{k^{\prime}, L, m}$ in the frame. The choice of a basis set or gauge $g^{\prime}$ fixes the states of $S_{j, k^{\prime}, L, m}$ that are values of rational numbers. These states are represented as $|\gamma, s\rangle_{j, k^{\prime}, L, m, g^{\prime}}$. Often the $L, m, g^{\prime}$ subscripts on the states will be dropped as they will not be needed for the discussion.

The description of the hybrid systems as strings of qukits is one of several possible

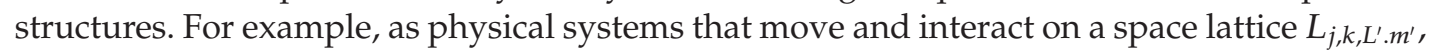
the strings could be open with free ends or closed loops. In this case, aspects of string theory [35] may be useful in describing the physics of the strings.

Whatever structure the hybrid systems have, it would be expected that, as bound systems, they have a spectrum of energy eigenstates described by some Hamiltonian $H_{j, k^{\prime}, L, m}^{S}$. If the rational number states of the hybrid system, $S_{j, k^{\prime}, L, m}$, are energy eigenstates, then one has the eigenvalue equation

$$
H_{j, k^{\prime}, L, m}^{S}|\gamma, s\rangle_{j, k^{\prime}, L, m}=E(\gamma, s)_{j, k^{\prime}, L, m}|\gamma, s\rangle_{j, k^{\prime}, L, m^{\prime}}
$$

where $E(\gamma, s)_{j, k^{\prime}, L, m}$ is the energy eigenvalue of the state $|\gamma, s\rangle_{j, k^{\prime}, L, m}$. The superscript $S$ on $H_{j, k^{\prime}, L, m}^{S}$ allows for the possibility that the Hamiltonian depends on the type of hybrid system. 
The gauge variable has been removed because the requirement that (4.1) is satisfied for some choice of $H_{j, k^{\prime}, L, m^{\prime}}^{S}$ fixes the gauge or basis to be the eigenstates of $H_{j, k^{\prime}, L, m}^{S}$. Since $H_{j, k^{\prime}, L, m}^{S}$ is not known, neither is the dependence of $E(\gamma, s)_{j, k^{\prime}, L, m}$ on $\gamma, s$.

The existence of a Hamiltonian for the $S$ hybrid systems means that there is energy associated with the values of rational numbers represented as states of hybrid systems. From this it follows that there are potentially many different energies associated with each rational number value. This is a consequence of the fact that each rational number value has many string state representations that differ by the number of leading and trailing $0 s$.

One way to resolve this problem is to let the energy of a hybrid system state with no leading or trailing $0 s$ be the energy value for the rational number represented by the state. In this way one has, for each $k$, a unique energy associated with the value of the rational number shown by the state.

One consequence of this association of energy to rational number values is that to each Cauchy sequence of rational number states of hybrid systems there corresponds a sequence of energies. The energy of the $n t h$ state in the sequence is given by $\left\langle\gamma_{n}, s_{n}\left|H_{j, k^{\prime}, L_{n}, m_{n}}\right| \gamma_{n}, s_{n}\right\rangle_{j, k^{\prime}, L_{n}, m_{n}}$.

It is not known at this point if the sequence of energies associated with a Cauchy sequence of hybrid system states converges or not. Even if energy sequences converge for Cauchy sequences in an equivalence class, the question remains whether or not the energy sequences converge to the same limit for all sequences in the equivalence class.

The above description is valid for one hybrid system. In order to describe more than one of these systems, another parameter, $h$, is needed whose values distinguish the states of the different $S_{j, k^{\prime}, L, m}$ systems. To this end the states $|\gamma, s\rangle_{j, k^{\prime}, L, m, g}$ of a system are expanded by including a parameter $h$ as in $|\gamma, h, s\rangle_{j, k^{\prime}, L, m, g}$. In this case the state of two $S_{j, k^{\prime}, L, m}$ is given by

$$
\left|\gamma_{1}, h_{1}, s_{1},\right\rangle_{j, k^{\prime}, L, m, g^{\prime}}\left|\gamma_{2}, h_{2}, s_{2}\right\rangle_{j, k^{\prime}, L, m, g^{\prime}}
$$

where $h_{1} \neq h_{2}$. This allows for the states of the two systems to have the same $\gamma$ and $s$ values.

Pairs of hybrid systems are of special interest because states of these pairs correspond to values of complex rational numbers. The state of one of the pairs is the real component and the other is the imaginary component. Since these components have different mathematical properties, the corresponding states in the pairs of states of hybrid systems must be distinguished in some way.

One method is to distinguish the hybrid systems in the pairs by an index $r, i$ added to $S_{j, k, L, m}$ as in $\left(S_{r}, S_{i}\right)_{j, k^{\prime}, L, m}$. In this case states of $S_{r}$ and $S_{i}$ are values of the real and imaginary components of rational numbers. In this case complex numbers are Cauchy sequences of states of pairs, $\left(S_{r}, S_{i}\right)_{j, k^{\prime}, L_{n}, m_{n}}$ of hybrid systems.

As might be expected, the kinematics and dynamics of hybrid systems $S_{j, k^{\prime}, L, m}$ in a frame $F_{j, k, g}$ are described relative to a space and time lattice in the frame. For example, a Schrödinger equation description of two hybrid systems interacting with one another is given by

$$
i \Delta_{t}^{f} \psi(t)=H \psi(t)
$$

$\Delta_{t}^{f}$ is the discrete forward time derivative where $\Delta_{t}^{f} \psi(t)=(\psi(t+\Delta)-\psi(t)) / \Delta$. Here $\psi(t)$ is the state of the two hybrid systems at time $t$. 
The Hamiltonian can be expressed as the sum of a Hamiltonian for the separate systems and an interaction part as in

$$
H=H_{0}+H_{\text {int }}
$$

For two hybrid systems $H_{0}=\sum_{i=1}^{2} H_{0, i}$ where

$$
H_{0, i}=\frac{-\hbar^{2}}{2 m_{S_{j, k^{\prime}, L, m}}} \Delta_{i}^{f} \cdot \Delta_{i}^{b}+H_{i, j, k^{\prime}, L, m}
$$

The first term of $H_{0, i}$ is the kinetic energy operator for the $i$ th $S_{j, k^{\prime}, L, m}$ system. The second term is the Hamiltonian for the internal states of the system. It is given by (4.1). Also $m_{S_{i, k^{\prime}, L, m}}$ is the mass of $S_{j, k^{\prime}, L, m}$. Also $\Delta^{f}$ and $\Delta^{b}$ are the discrete forward and backward space derivatives, and $\hbar$ is Planck's constant. The dot product indicates the usual sum over the product of the $D$ components in the derivatives. Note that a possible dependence of the mass of $S_{j, k^{\prime}, L, m}$ on $k^{\prime}, L, m$ has been included.

The question arises regarding how one should view $N$-tuple hybrid systems as physical systems. Should they be regarded as $N$ independent systems each with its own Hamiltonian $\left(H_{\text {int }}=0\right.$ in (4.4) $)$ or as systems bound together with energy eigenstates that are quite different from those of the single hybrid systems in isolation?

One way to shed light on this question is to examine physical representations of number tuples in computers. Their $N$-tuples of numbers are represented as $N$ strings of bits or of qubits (spin $1 / 2$ systems) bound to a background matrix of potential wells where each well contains one qubit. The locations of the qubits in the background matrix determines their assembly into strings and into tuples of strings.

Here it is assumed that $N$ tuples of hybrid systems consist of $N S_{j, k^{\prime}, L, m}$ systems bound together in some fashion. Details of the binding and its effect on the states of the individual $S_{j, k^{\prime}, L, m}$ in the $N$-tuple are not known at this point. However it will be assumed that the effect is negligible. In this case the energy of each component state $\left|\gamma_{z}, h_{z}, s_{z}\right\rangle$ in the $N$ tuple will be assumed to be the same as that for an individual $S_{j, k^{\prime}, L, m}$ system. Then, the energy of the state $|\bar{\gamma}, \bar{h}, \bar{s}\rangle$ is the sum of the energies of the individual component states. Also the energy is assumed to be independent of the $h$ values.

This picture is supported by the actual states of computers and their computations. The background potential well matrix that contains the $N$-tuples of qubit string states is tied to the computer. Since the computer itself is a physical system, it can be translated, rotated, or given a constant velocity boost. In all these transformations the states of the qubit strings in the $N$ tuples and the space relations of the $N$ qubit strings to one another are unchanged. These parameters would be changed if two computers collided with one another with sufficient energy to disrupt the internal workings.

This picture of each frame containing physical systems and a plethora of different hybrid systems and their tuples may seem objectionable. However, one should recall that here one is working in a possible domain of a coherent theory of physics and mathematics together. In this case the domain might be expected to include many types of hybrid systems that have both physical and mathematical properties. This is in addition to the presence of physical systems and mathematical systems. 


\section{Frame Entities as Viewed in a Parent Frame}

So far it has been seen that each frame, $F_{j, k, g}$, in the frame field contains a set $\mathcal{L}_{j, k}$ of space and time lattices where the number of points in each dimension and the point spacing satisfy (3.1) for some $L$ and $m$. The frame also contains qukit string systems as hybrid $S_{j, k^{\prime}, L^{\prime}, m^{\prime}}$ systems and various tuples of these systems. Here $k^{\prime}, L^{\prime}, m^{\prime}$ need have no relation to $k, L, m$. Each frame also contains physical theories as mathematical structures based on $R_{j, k, g}$ and $C_{j, k, g}$ the real and complex number base of the frame. These theories describe the kinematics and dynamics of physical systems on the space and time lattices in the frame. For quantum systems these theories include Hilbert spaces as vector spaces over $C_{j, k, g}$.

Since this is true for every frame, it is true for a frame $F_{j, k, g}$ and for a parent frame $F_{j-1, k^{\prime}, g^{\prime}}$. This raises the question of how entities in a frame are seen by an observer in a frame at an adjacent iteration stage. As was noted in Section 2.2, it is assumed that ancestor frames and their contents are not visible to observers in descendant frames; however, descendant frames and their contents are visible to observers in ancestor frames. It follows that observers in frame $F_{j, k, g}$ cannot see frame $F_{j-1, k^{\prime}, g^{\prime}}$ or any of its contents. However, observers in $F_{j-1, k^{\prime}, g^{\prime}}$ can see $F_{j, k, g}$ and its contents. (This restriction has to be relaxed for cyclic frame fields.)

One consequence of the relations between frames at different iteration stages is that entities in a frame, that are seen by an observer in the frame as featureless and with no structure, correspond to entities in a parent frame that have structure. For example, elements of $R_{j, k, g}, C_{j, k, g}$ are seen by an observer in $F_{j, k, g}$ as abstract, featureless objects with no properties other than those derived from the relevant axiom sets. However, to observers in a parent frame $F_{j-1, k^{\prime}, g^{\prime}}$, numbers in $R_{j, k, g}, C_{j, k, g}$ are seen as equivalence classes (pairs of equivalence classes for $C_{j, k, g}$ ) of Cauchy sequences of states of base $k$ qukit string systems. Thus entities that are abstract and featureless in a frame have structure as elements of a parent frame.

It is useful to represent these two in-frame views by superscripts $j$ and $j-1$. Thus $R_{j, k, g^{\prime}}^{j-1} C_{j, k, g^{\prime}}^{j-1}$ and $R_{j, k, g^{\prime}}^{j} C_{j, k, g}^{j}=R_{j, k, g}, C_{j, k, g}$ denote the stage $j-1$ and stage $j$ frame views of the number base of frame $F_{j, k, g}$. They are often referred to in the following as parent frame images of $R_{j, k, g}, C_{j, k, g}$.

The distinction between elements of a frame and their images in a parent frame exists for other frame entities as well. The state

$$
\psi_{j}=\sum_{\alpha} d_{j, \alpha}^{j}|\alpha\rangle_{j, k, g}^{j}
$$

in $\mathfrak{\ell}_{j, k, g}^{j}$ corresponds to the state

$$
\psi_{j}^{j-1}=\sum_{\alpha} d_{j, \alpha}^{j-1}|\alpha\rangle_{j, k, g}^{j-1}
$$

in $\mathscr{\ell}_{j, k, g^{\prime}}^{j-1}$, which is the parent frame image of $\mathscr{H}_{j, k, g}$. In the above $d_{j, \alpha}^{j}$ is a featureless abstract complex number in $C_{j, k, g}$ whereas $d_{j, \alpha}^{j-1}$, as an element of $C_{j, k, g}^{j-1}$, is an equivalence class of Cauchy sequences of hybrid system states.

The use of stage superscripts and subscripts applies to other frame entities, such as hybrid systems, physical systems, and space and time lattices. A hybrid system $S_{j, k^{\prime}, L^{\prime}, m^{\prime}}$ in 
$F_{j, k, g}$ has $S_{j, k^{\prime}, L^{\prime}, m^{\prime}}^{j-1}$ as a parent frame image. States $\left|\gamma^{\prime}, s^{\prime}\right\rangle_{j}$ of $S_{j, k^{\prime}, L^{\prime}, m^{\prime}}$ are vectors in the Hilbert space $\mathscr{l}_{j, k, g}$, in $F_{j, k, g}$. States $\left|\gamma^{\prime}, s^{\prime}\right\rangle_{j}^{j-1}$ of $S_{j, k^{\prime}, L^{\prime}, m^{\prime}}^{j-1}$ are vectors in $\mathfrak{l}_{j, k, g}^{j-1}$. The two states are different in that $\left|\gamma^{\prime}, s^{\prime}\right\rangle_{j}$ is an eigenstate of an operator whose corresponding eigenvalue $\gamma^{\prime}, s^{\prime}$ is a rational real number with no structure.

The state $\left|\gamma^{\prime}, s^{\prime}\right\rangle_{j}^{j-1}$ of $S_{j, k^{\prime}, L^{\prime}, m^{\prime}}^{j-1}$ is an eigenstate of a number value operator whose corresponding eigenvalues are rational real numbers in $R_{j, k, g}^{j-1}$ corresponding to $\left(\gamma^{\prime}, s^{\prime}\right)$. These eigenvalues are equivalence classes of Cauchy sequences of states of hybrid systems $S_{j-1, k, L, m}$ in a $j-1$ stage frame. Here $k, j-1$ are fixed and $L=L_{n}$ and $m=m_{n}$ for the $n$th term in the sequence. This accounts for the fact that each state in the sequence is a state of a different hybrid system with $j-1, k$ held fixed.

The eigenvalue equivalence class is a base $k^{\prime}$ real rational number. Since it is an element of $R_{j, k, g^{\prime}}^{j-1}$ it contains a constant sequence of hybrid system states if and only if all prime factors of $k^{\prime}$ are factors of $k$. If this is the case, then one can equate the equivalence class to a single state of a hybrid system to conclude that the eigenvalue associated with $\left|\gamma^{\prime}, s^{\prime}\right\rangle_{j}^{j-1}$ is a state of a hybrid system $S_{j-1, k, L, m}$ in stage $j-1$ frame.(Note that the language used here avoids referring to the absolute existence of systems with properties independent of the frames. The emphasis is on the view of systems in different frames. Thus $b_{j}=b_{j}^{j}$ is the view of a physical system $b$ as seen by an observer in a stage $j$ frame. The image of this view in a stage $j-1$ frame is denoted by $b_{j}^{j-1}$. The difference between the two is that physical properties of $b_{j}$, as eigenvalues of operators over $\mathfrak{d}_{j, k, g}$, are featureless abstract real numbers. Properties of $b_{j}^{j-1}$, as operator eigenvalues, are equivalence classes of Cauchy sequences of hybrid system states. A frame independent description would be expected to appear only asymptotically when the frames in the field are merged.)

If $k^{\prime}$ has prime factors that are not prime factors of $k$ (such as $k^{\prime}=6$ and $k=3$ ), the eigenvalue for the eigenstate $\left|\gamma^{\prime}, s^{\prime}\right\rangle_{j}^{j-1}$ is still a real rational number. However, as an equivalence class of Cauchy sequences of base $k$ hybrid system states, (which it must be as a real number in $C_{j, k, g}^{j-1}$ ) it does not contain a constant sequence of hybrid system states. Instead it contains a sequence that corresponds to an infinite repetition of a base $k$ hybrid system state (just like the decimal expansion of $1 / 6=0.16666 \ldots$ ).

\subsection{Parent Frame Views of Lattice Point Locations}

A similar representation holds for parent frame views of point locations of lattices. Let $L_{j, k, L, m}$ denote a lattice of $D$ space dimensions and one time dimension in a frame $F_{j, k, g}$. The components $x_{j, z}$ (with $z=1, \ldots, D$ ) of the $D$ space locations $\bar{x}_{j}$ of the lattice points, $p_{j}$, are such that $x_{j, z}$ is a rational real number. The lattice points are abstract and have no structure other than that imparted by the values $\bar{x}_{j}$. As rational real numbers in $R_{j, k, g}$, the $x_{j, z}$ have no structure other than the requirement that they are both rational and real numbers.

The view or image of $L_{j, k, L, m}$ from the position of an observer in a stage $j-1$ parent frame is denoted by $L_{j, k, L, m}^{j-1}$. The image points and locations of points in $L_{j, k, L, m}^{j-1}$ are denoted by $p_{j}^{j-1}$ and $\bar{x}_{j}^{j-1}$.

The space point locations $\bar{x}_{j}^{j-1}$ are different from the $\bar{x}_{j}$ in that they have more structure. This follows from the fact that they are $D$ tuples of rational real numbers in $R_{j, k, g}^{j-1}$. It follows 
from this that each component, $x_{j, z}^{j-1}$, of a space point location, $\bar{x}_{j}^{j-1}$, in $L_{j, k, L, m}^{j-1}$ is an equivalence class of Cauchy sequences of states of hybrid systems. Since each of the $D$ equivalence classes is a real number equivalent of a rational number value, the equivalence class includes many (numerically) constant sequences of hybrid system states. The existence of constant sequences follows from the observation that the $k$ subscript of the lattice image is the same as that for $R_{j, k, g}^{j-1}$. The states in the different sequences differ by the presence of different numbers of leading and trailing $0 s$.

A useful way to select a unique constant sequence is to require that all states in the sequence be a unique state of the hybrid system $S_{j-1, k, L, m}$ where the $k, L, m$ subscripts are the same as those for $L_{j, k, L, m}^{j-1}$. Replacement of the sequence by its single component state gives the result that, for each $z, x_{j, z}^{j-1}$ is a state of $S_{j-1, k, L, m}$.

It follows from this that the locations of space points $p_{j}$ in $L_{j, k, L, m}$, as viewed from a stage $j-1$ frame, are seen as states of a $D$-tuple $S_{j-1, k, L, m}^{D}$ of hybrid systems in the stage $j-1$ frame. These states correspond to $D$-tuples of rational number values.

A similar representation of the time points in the lattices is possible for the real rational number time values. In this case the time values of a lattice are seen in a parent frame as rational number states of a hybrid system.

\section{Lattices and Hybrid Systems}

The above description of a stage $j-1$ frame view of lattices gives point locations of $L_{j, k, L, m}^{j-1}$ as states of a $D$ tuple of hybrid systems $S_{j-1, k, L, m}^{x, D}$ for the space part and states of another hybrid system $S_{j-1, k, L, m}^{t}$ for the time part. The superscripts $s$ and $t$ denote the hybrid systems associated with space and time points, respectively.

This strongly suggests that each space point image $p_{j}^{x, j-1}$ in the space part of a parent frame image, $L_{j, k, L, m^{\prime}}^{j-1}$ of $L_{j, k, L, m}$ should be identified with a $D$ tuple, $S_{j-1, k, L, m}^{x, D}$ of hybrid systems and each time point image $p_{j}^{t, j-1}$ in the time part should be identified with a single hybrid system, $S_{j-1, k, L, m}^{t}$. For each image point $p_{j}^{x, j-1}$, the location is given by the state of the $D$ tuple $S_{j-1, k, L, m^{\prime}}^{x, D}$ of hybrid systems in a parent frame that is associated with the space point image. Similarly the location of each image time point in $L_{j, k, L, m}^{j-1}$ is given by the state of the hybrid system, $S_{j-1, k, L, m^{\prime}}^{t}$ associated with the point.

This shows that set of all parent frame images of the $k^{L D}$ space points of $L_{j, k, L, m}$ become a set of $k^{L D} D$ tuples of parent frame hybrid systems, $S_{j-1, k, L, m^{\prime}}^{x, D}$ with the state of each $D$ tuple corresponding to the image space point location in $L_{j, k, L, m}^{j-1}$. The parent frame images of the $k^{D}$ time points of $L_{j, k, L, m}$ become a set of $k^{L}$ hybrid systems $S_{j-1, k, L, m}^{t}$. Each is in a different state corresponding to the different possible lattice time values.

Figure 2 illustrates the situation described above for lattices with one space and one time dimension. The stage $j$ and $j-1$ lattice points are shown by the intersection of the grid lines. For the stage $j$ lattice the points correspond to rational number pairs whose locations are given by the values of the pairs of numbers. For the stage $j-1$ image lattice the points correspond to pairs of hybrid systems, one for the space dimension and one for the time dimension. Point locations are given by the states of the hybrid system pairs. Non relativistic world paths for physical systems $b_{j}$ and its stage $j-1$ image $b_{j}^{j-1}$ are also shown. 


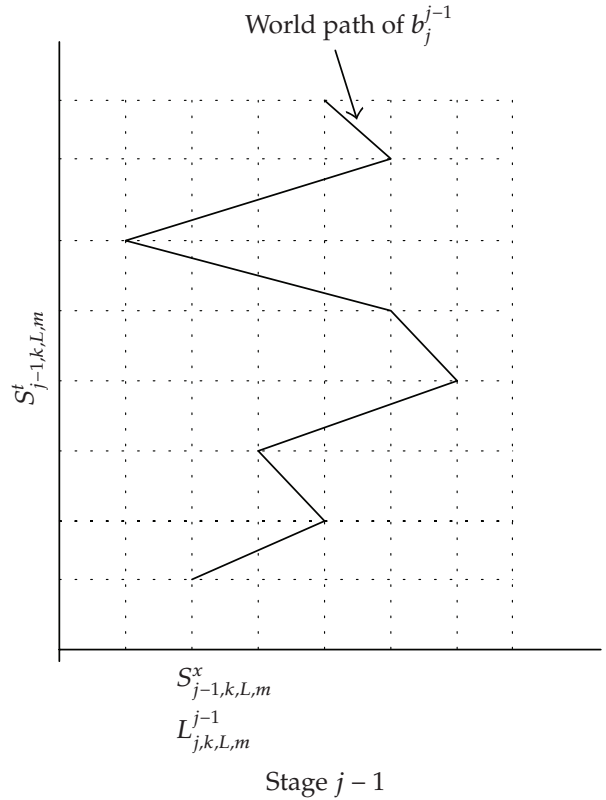

(a)

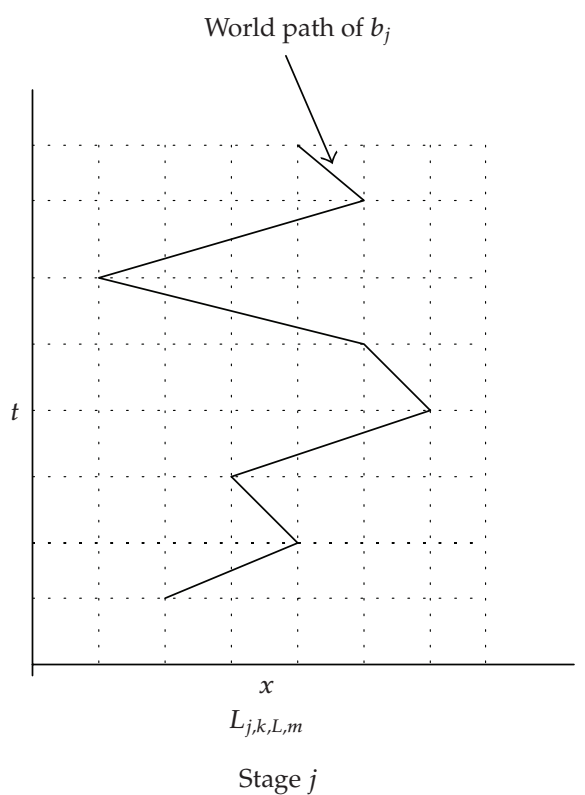

(b)

Figure 2: Stage $j$ and stage $j-1$ images of one-dimensional space and time lattices. Lattice points are indicated by intersections of lines in the two-dimensional grid. In $L_{j, k, L, m}$ the points $p_{j}$ consist of pairs of rational numbers. In $L_{j, k, L, m}^{j-1}$ the image points $p_{j}^{j-1}$ consist of pairs $S_{j-1, k, L, m^{\prime}}^{x} S_{j-1, k, L, m}^{t}$ of hybrid systems with point locations given by the states of the system pairs. Nonrelativistic world paths of a stage $j$ physical system $b_{j}$ and of its stage $j-1$ image $b_{j}^{j-1}$ are shown as solid lines. Note that $b$ can also be a hybrid system.

It is of interest to compare the view here with that in [8]. Tegmark's explicitly stated view is that real numbers, as labels of space and time points, are distinct from the points themselves. This is similar to the setup here in that points of parent frame images of lattices are tuples of hybrid systems, and locations or labels of the points of parent frame images of lattice are states of tuples of hybrid systems.

The differing views of hybrid systems as either number systems or physical systems may seem strange when viewed from a perspective outside the frame field and in the usual physical universe. However it is appropriate for a coherent theory of physics and mathematics together as such a theory might have systems that represent different entities, depending on how they are viewed.

\subsection{Energy of Space Points in $L_{j, k, L, m}^{j-1}$}

The description of parent frame images of lattice space points and their locations as $D$ tuples of hybrid systems and states of the tuples means that the image of each point has a mass. The mass is equal to that of the $D$ tuple of hybrid systems associated with each point image. The (rest) masses of all space points in an image lattice $L_{j, k, L, m}^{j-1}$ should be the same as the $D$ tuples of hybrid systems associated with each point are the all the same. However each of 
the tuples is in a different image state $\bar{x}_{j}^{j-1}$ that corresponds to the different locations of each point image.

Each component $x_{j, z}^{j-1}$ of $\bar{x}_{j}^{j-1}$ corresponds to a hybrid system state $\left|+, s_{z}\right\rangle_{j}^{j-1}$ of a component hybrid system $S_{z, j-1, k, L, m}^{D}$ in the $D$ tuple (note the subscript $z$ ). Each of these component hybrid system states is an energy eigenstate of a hybrid system Hamiltonian. The corresponding energy eigenvalue, $E\left(+, s_{z}\right)_{j-1}$, is defined by (4.1). Here $\gamma$ is positive as the lattice component locations are all $\geq 0$.

If the component hybrid systems in a $D$ tuple do not interact with one another, then the energy associated with a parent frame image lattice location is the sum of the component energies. In this case the energy associated with the location image, $|+, \bar{s}\rangle_{j}^{j-1}$, of $\bar{x}_{j}$ is given by

$$
E(+, \bar{s})_{j-1}=\sum_{z=1}^{D} E\left(+, s_{z}\right)_{j-1}
$$

Here $|+, \bar{s}\rangle_{j}^{j-1}$ denotes the tensor product of all $D$ component states $\left|+, s_{z}\right\rangle_{j}^{j-1}$. If the component systems in a $D$ tuple do interact with one another, then the situation becomes more complex as the Hamiltonian and energy eigenvalues must take account of the interactions.

At this point the specific dependence of the energy on the parent frame image of the lattice point locations is not known as it depends on the properties of the hybrid system Hamiltonian. Nevertheless the existence of energies associated with locations of points of parent frame images of a space lattice is intriguing. One should note, though, that this association of energy to space points holds only for parent frame images. It does not extend to lattices $L_{j, k, L, m}$ when viewed by an observer in $F_{j, k, g}$.

This aspect is one reason why one needs to do more work, particularly on the merging of frames in the frame field. It is quite possible that, in the case of a cyclic frame field, some aspects of the association of energy with space points in the same frame will be preserved. Note that for cyclic frame fields the restriction that an observer cannot see ancestor frames or their contents must be relaxed. The reason is that ancestor frames are also descendant frames.

\subsection{Dynamics of Systems as Seen from a Parent Frame}

The description of the motion of hybrid systems and other physical systems in a stage $j$ frame, as seen from a parent stage $j-1$ frame, is interesting. The reason is that the dynamics and kinematics of systems are based on a parent frame image lattice, $L_{j, k, L, m}^{j-1}$, whose space points and point locations are $D$ tuples, $S_{j-1, k, L, m^{\prime}}^{x, D}$ and tensor product states $\bigotimes_{z=1}^{D}\left|+, s_{z}\right\rangle_{j-1}$ of the $D$ tuples of hybrid systems. The time points and point locations are hybrid systems, $S_{j-1, k, L, m^{\prime}}^{t}$ and states of these systems. The $x$ and $t$ superscripts allow for the possibility that the hybrid systems associated with space point images may be different from those associated with time images.

It follows that the stage $j-1$ frame description of the motion and dynamics of stage $j$ systems is described relative to the states of certain hybrid systems in the parent frame. To understand this consider a simple example where $\Psi(x, t)_{j}$ denotes the state of some physical system $b_{j}$ in a stage $j$ frame at position $x$ and at time $t$. The pair $x, t$ denote a point in a stage $j$ frame space and time lattice $L_{j, k, L, m}$ which, for simplicity, consists of one space and one 
time dimension. The stage $j$ frame time evolution of the state $\Psi(x, t)_{j}$ is given by a discrete Schrödinger Equation:

$$
i \hbar \Delta_{t, j}^{f} \Psi_{j}(x, t)=H_{j} \Psi_{j}(x, t)
$$

Here $\Delta_{t, j}^{f}$ is the discrete forward time derivative defined by

$$
\Delta_{t, j}^{f} \Psi_{j}(x, t)=\frac{\Psi_{j}\left(x, t+\Delta_{j}\right)-\Psi_{j}(x, t)}{\Delta_{j}}
$$

As a sum of kinetic and potential terms, the Hamiltonian, $H_{j}$, has the form

$$
H_{j}=-\frac{\hbar^{2} \Delta_{j}^{f} \Delta_{j}^{b}}{2 m_{b_{j}}}+V_{j} .
$$

To keep things simple, the description is restricted to just one $b_{j}$ system interacting with an external potential. In this case $V_{j}=V_{j}(x)$. Also $\hbar$ is Planck's constant and $m_{b_{j}}$ is the mass of system $b_{j}$.

In the above, the forward and backward discrete derivatives $\Delta_{j}^{f}$ and $\Delta_{j}^{b}$ are defined similar to the forward time derivative. One has

$$
\begin{aligned}
& \Delta_{j}^{f} \Psi_{j}(x, t)=\frac{\Psi_{j}\left(x+\Delta_{j}, t\right)-\Psi_{j}(x, t)}{\Delta_{j}}, \\
& \Delta_{j}^{b} \Psi_{j}(x, t)=\frac{\Psi_{j}\left(x-\Delta_{j}, t\right)-\Psi_{j}(x, t)}{\Delta_{j}} .
\end{aligned}
$$

This description of the time development and Hamiltonian for a system $b_{j}$ is a description in a stage $j$ frame. Viewed from a parent stage $j-1$ frame the image of the Schrödinger equation, (6.2) describes the motion of the system $b$ in the image lattice $L_{j, k, L, m}^{j-1}$. Since the space and time point locations in the image lattice are states of hybrid systems $S_{j-1, k, L, m}^{x}$ and $S_{j-1, k, L, m}^{t}$ the image Schrödinger equation describes the motion of system $b$ relative to these states. The image equation is given by

$$
i \hbar\left(\Delta^{f}\right)_{j, s_{t}}^{j-1} \Psi_{j}^{j-1}\left(s_{x}, s_{t}\right)=H_{j}^{j-1} \Psi_{j}^{j-1}\left(s_{x}, s_{t}\right)
$$

The image state, $\Psi_{j}^{j-1}\left(s_{x}, s_{t}\right)$, is the same state in the Hilbert space $\mathfrak{l}_{j, k, g}^{j-1}$ as $\Psi_{j}(x, t)$ is in $\mathfrak{l}_{j, k, g}$. Here $s_{x}$ and $s_{t}$ are shorthand notations for the hybrid system states, $\left|+, s_{x}\right\rangle_{j-1}$ and $\left|+, s_{t}\right\rangle_{j-1}$ of $S_{j-1, k, L, m}^{x}$ and $S_{j-1, k, L, m}^{t}$. 
The Hamiltonian $H_{j}^{j-1}$ is given by

$$
H_{j}^{j-1}=-\frac{\hbar^{2}\left(\Delta^{f}\right)_{j, s_{x}}^{j-1}\left(\Delta^{b}\right)_{j, s_{x}}^{j-1}}{2 m_{b_{j}^{j-1}}}+V_{j}^{j-1}\left(s_{x}\right) .
$$

The potential $V_{j}^{j-1}\left(s_{x}\right)$ is a function of the states $\left|+, s_{x}\right\rangle_{j-1}$ of $S_{j-1, k, L, m}^{x}$. The value of $m_{b_{j}^{j-1}}$ is a real number in $R_{j, k, g}^{j-1}$ that is expected to be the same as that of $m_{b_{j}^{j}}$ in $R_{j, k, g}^{j}$.

The forward and backward discrete derivatives are expressed by equations similar to (6.5):

$$
\begin{aligned}
& \left(\Delta^{f}\right)_{j, s_{x}}^{j-1} \Psi_{j}^{j-1}\left(s_{x}, s_{t}\right)=\frac{\Psi_{j}^{j-1}\left(s_{x}+1, s_{t}\right)-\Psi_{j}^{j-1}\left(s_{x}, s_{t}\right)}{\Delta_{j}^{j-1}}, \\
& \left(\Delta^{b}\right)_{j, s_{x}}^{j-1} \Psi_{j}^{j-1}\left(s_{x}, s_{t}\right)=\frac{\Psi_{j}^{j-1}\left(s_{x}-1, s_{t}\right)-\Psi_{j}^{j-1}\left(s_{x}, s_{t}\right)}{\Delta_{j}^{j-1}} .
\end{aligned}
$$

In these equations $s_{x}+1$ and $s_{x}-1$ denote the hybrid system states $\left|+, s_{x}+{ }_{A} 1\right\rangle_{x}$ and $\left|+, s_{x}-{ }_{A} 1\right\rangle_{x}$ where the subscript $A$ denotes arithmetic addition and subtraction. For example, if $\left|+, s_{x}\right\rangle_{x}=$ $|100+111\rangle_{x}$ in binary then, $\left|+, s_{x}+{ }_{A} 1\right\rangle_{x}=|101+000\rangle_{x}$.

This use of states of $S_{j-1, k, L, m}^{x}$ and $S_{j-1, k, L, m}^{t}$ as point locations of an image space and time lattice must be reconciled with the observation that these hybrid systems are dynamical systems that move and interact with one another and with other physical systems. If the $S_{j-1, k, L, m}^{x}$ and $S_{j-1, k, L, m}^{t}$ and their states are to serve as points and point locations of a space and time lattice image, they must be dynamically very stable and resistant to change. This suggests that these systems must be very massive and that their interactions with each other and other systems are such that state changes occur very rarely, possibly on the order of cosmological time intervals. (The dynamics of these systems on space and time lattices $L_{j-1, k^{\prime}, L^{\prime}, m^{\prime}}$ is described in a stage $j-1$ frame. Here $k^{\prime}, L^{\prime}, m^{\prime}$ need have no relation to $k, L, m$.)

The reason for these restrictions on the properties of space and time hybrid systems is that one expects the space and time used to describe motion of systems to be quite stable and to change at most very slowly. Changes, if any, would be expected to be similar to those predicted by the Einstein equations of general relativity.

\section{Discussion}

It is to be emphasized that the work presented so far is only a beginning to the development of a complete framework for a coherent theory of physics and mathematics together. Not only that but one must also find a way to reconcile the multiplicity of universes, one for each frame, to the view that there is only one physical universe.

One way to achieve reconciliation is to drop the single universe view and to relate the multiplicity of frame representations of physics and mathematics to the different many physical universes views of physics. These include physical universes in existing in different bubbles of space time [36, 37] and other descriptions of multiple universes $[4,38-40]$ 
including the Everett Wheeler description [41]. Whether any of these are relevant here or not will have to await future work.

If one sticks to the single physical universe view, then the frames with their different universes and space and time representations need to be merged or collapsed to arrive in some limit at the existing physical universe with one space time. This applies in particular to the iteration stage and gauge degrees of freedom as their presence is limited to the quantum representation of numbers.

One expected consequence of the merging is that it will result in the emergence of a single background space time as an asymptotic limit of the merging of the space time lattices in the different frames. Whether the ultimate space time background is a continuum, a foam [28-31], or has some other form, should be determined by details of the merging process.

In addition the merging may affect other entities in the frames. Physical systems, denoted collectively as $b_{j}$, may become the observed physical systems in the space time background. In addition, hybrid systems may split into either physical systems or mathematical systems.

One potentially useful approach to frame merging is the use of gauge theory techniques $[17,42]$ to merge frames in different iteration stages. One hopes that some aspects of the standard model [43] in physics will be useful here. This will require inclusion of relativistic treatment of systems and quantum fields in the frames.

This look into a possible future approach emphasizes how much there is to accomplish. Nevertheless one may hope that the work presented here is a beginning to the development of a coherent theory. The expansion of the frames in the frame field to include, not only mathematical systems but also space and time lattices, and hybrid systems that are both mathematical systems and physical systems, seems reasonable from the viewpoint of a coherent theory of physics and mathematics together. One might expect such a theory to contain systems that can be either physical systems or mathematical systems.

The use of massive hybrid systems to be stage $j-1$ frame images of points of space and time lattices in stage $j$ frames suggests that there must be different types of hybrid systems. For example, stage $j$ theoretical predictions of the values of some physical quantity $Q$ are, in general, real numbers in $R_{j, k, g}$. Their images in a stage $j-1$ parent frame are equivalence classes of Cauchy sequences of states of hybrid systems $S_{j-1, k, L, m}$ where $L, m$ are dependent on positions in the Cauchy sequences. (If one introduces limit hybrid systems containing an infinite number of qubits, then an equivalence class of Cauchy sequences could be replaced by just one limit system whose state corresponds to an infinite sequence of digits that is a canonical representative of the class.) If the predicted values are rational numbers expressible as a finite string of base $k$ digits, then the stage $j-1$ values can be expressed as states of $S_{j-1, k, L, m}$ rather than equivalence classes of sequences of states. If the images of properties of the physical quantities $Q$ are to be reflected in the properties of the hybrid systems, then different types of hybrid systems, such as $S_{j-1, k, L, m^{\prime}}^{Q}$ must be associated with different physical quantities.

Whether these descriptions of parent frame images as hybrid systems will remain or will have to be modified remains to be seen. However, it should be recalled that these images are based on the dual role played by values of rational numbers both as mathematical systems and as locations of components of points in the lattices. Recall that the notion of a point in a lattice is separated from the location of the point just as the notion of a number, as a mathematical system, is separated from the value of a number. This use of number and number value is different from the usual use in mathematics in that expressions, as 135.79, are usually considered as rational numbers and not as values of rational numbers. Here a rational 
number, as a hybrid system, is similar to the usual mathematical concept of a set of rational numbers as a model of the rational number axioms.

In conclusion, it is worth reiterating the last paragraphs at the end of the introduction. Whatever one thinks of the ideas presented in the paper, the following points should be kept in mind. Two of the three dimensions of the field of reference frames are present only for quantum theory representations of the real and complex numbers. These are the gauge or basis degree of freedom and the iteration stage degree of freedom. They are not present in classical descriptions. The number base degree of freedom is present for both quantum and classical representations based on rational number representations by digit strings.

The presence of the gauge and iteration degrees of freedom in the quantum representation described here is independent of the description of rational number values as states of qukit string systems. Any quantum representation of the rational numbers, such as states $|l, n\rangle$ of integer pairs, where the states are elements of a vector space will result in a frame field with gauge and iteration degrees of freedom.

Finally, the importance of numbers to physics and mathematics should be emphasized. It is hoped that more work on combining quantum physics and the quantum theory of numbers will be done. The need for this is based on the observation that natural numbers, integers, rational numbers, and probably real and complex numbers are even more fundamental to physics than is geometry.

\section{Acknowledgment}

This work was supported by the U.S. Department of Energy, Office of Nuclear Physics, under Contract no. DE-AC02-06CH11357.

\section{References}

[1] E. Wigner, "The unreasonable effectiveness of mathematics in the natural sciences," Communications in Pure and Applied Mathematics, vol. 13, no. 1, 1960, Reprinted in Symmetries and Reflections, pp. 222-237, Indiana University Press, Bloomington, Ind, USA, 1966.

[2] R. W. Hamming, "The unreasonable effectiveness of mathematics," The American Mathematical Monthly, vol. 87, no. 2, pp. 81-90, 1980.

[3] G. Boniolo, P. Budinich, and M. Trobok, Eds., The Role of Mathematics in Physical Sciences: Interdisciplinary and Philosophical Aspects, Springer, Dordrecht, The Netherlands, 2005.

[4] P. Davies, The Goldilocks Enigma, Mifflin, New York, NY, USA, 2006.

[5] D. Bendaniel, "Linking the foundations of physics and mathematics," http://arxiv.org/abs/math$\mathrm{ph} / 9907004$.

[6] S. Wolfram, A New Kind of Science, Wolfram Media, Champaign, Ill, USA, 2002.

[7] M. Tegmark, "Is "the theory of everything" merely the ultimate ensemble theory?" Annals of Physics, vol. 270, no. 1, pp. 1-51, 1998.

[8] M. Tegmark, "The mathematical universe," Foundations of Physics, vol. 38, no. 2, pp. 101-150, 2008.

[9] A. N. Bernal, M. Sánchez, and F. J. S. Gil, "Physics from scratch. Letter on M. Tegmark's "The Mathematical Universe"," http://arxiv.org/abs/0803.0944.

[10] P. Benioff, "Towards a coherent theory of physics and mathematics," Foundations of Physics, vol. 32, no. 7, pp. 989-1029, 2002.

[11] P. Benioff, "Towards a coherent theory of physics and mathematics: the theory-experiment connection," Foundations of Physics, vol. 35, no. 11, pp. 1825-1856, 2005.

[12] P. Benioff, "A representation of real and complex numbers in quantum theory," International Journal of Pure and Applied Mathematics, vol. 39, no. 3, pp. 297-339, 2007.

[13] P. Benioff, "Properties of frame fields based on quantum theory representations of real and complex numbers," in Advances in Quantum Computation, K. Mahdavi and D. Doslover, Eds., vol. 482 of Contemporary Mathematics, pp. 125-163, University of Texas, Tyler, Tex, USA, 2007. 
[14] Y. Aharonov and T. Kaufherr, "Quantum frames of reference," Physical Review D, vol. 30, no. 2, pp. 368-385, 1984.

[15] S. D. Bartlett, T. Rudolph, and R. W. Spekkens, “Decoherence-full subsystems and the cryptographic power of a private shared reference frame," Physical Review A, vol. 70, Article ID 032307, 2004.

[16] S. J. van Enk, "Quantifying the resource of sharing a reference frame," Physical Review A, vol. 71, no. 3, Article ID 032339, 8 pages, 2005.

[17] S. J. van Enk, “Quantum communication, reference frames, and gauge theory," Physical Review A, vol. 73, no. 4, Article ID 042306, 9 pages, 2006.

[18] D. Poulin and J. Yard, "Dynamics of a quantum reference frame," New Journal of Physics, vol. 9, article $156,2007$.

[19] S. D. Bartlett, T. Rudolph, and R. W. Spekkens, “Reference frames, superselection rules, and quantum information," Reviews of Modern Physics, vol. 79, no. 2, pp. 555-609, 2007.

[20] R. Landauer, "Information is physical," Physics Today, vol. 44, no. 5, pp. 23-29, 1991.

[21] P. Benioff, "The representation of numbers in quantum mechanics," Algorithmica, vol. 34, no. 4, pp. 529-559, 2002.

[22] F. A. Raffa and M. Rasetti, “Natural numbers and quantum states in fock space," International Journal of Quantum Information, vol. 7, supplement, pp. 221-228, 2009.

[23] "The quantum structure of space and time," in Proceedings of the 23rd Solvay Conference, D. Gross, M. Henneaux, and A. Sevrin, Eds., World Scientific Press, Brussels, Belgium, December 2005.

[24] A. Ashtekar, "Quantum mechanics of geometry," http://arxiv.org/abs/gr-qc/9901023.

[25] J. Madore, "Noncommutative geometry for pedestrians," http:/ /arxiv.org/abs/gr-qc/9906059.

[26] A. Connes, Noncommutative Geometry, Academic Press, New York, NY, USA, 1994.

[27] G. T. Horowitz, "Spacetime in string theory," New Journal of Physics, vol. 7, article 201, 2005.

[28] Y. Jack Ng, "Holographic foam, dark energy and infinite statistics," Physics Letters B, vol. 657, no. 1-3, pp. 10-14, 2007.

[29] Y. Jack Ng, "Spacetime foam and dark energy," in AIP Conference, vol. 1115, pp. 74-79, Cairo, 2008, http://arxiv.org/abs/0808.1261.

[30] G. Amelino-Camelia, "A phenomenological description of space-time noise in quantum gravity," Nature, vol. 410, no. 6832, pp. 1065-1067, 2001.

[31] A. A. Kirillov and E. P. Savelova, "Spacetime foam," http://arxiv.org/abs/0806.0443.

[32] A. Ashtekar and J. Lewandowski, "Background independent quantum gravity: a status report," Classical and Quantum Gravity, vol. 21, no. 15, pp. R53-R152, 2004.

[33] N. Seiberg, "Emergent spacetime in the quantum structure of space and time," in Proceedings of the 23rd Solvay Conference, D. Gross, M. Henneaux, and A. Sevrin, Eds., World Scientific Press, Brussels, Belgium, December 2005.

[34] D. Kielpinski, C. Monroe, and D. J. Wineland, "Architecture for a large-scale ion-trap quantum computer," Nature, vol. 417, no. 6890, pp. 709-711, 2002.

[35] B. Zwiebach, A First Course in String Theory, Cambridge University Press, Cambridge, UK, 2004.

[36] A. D. Linde, Inflation and Quantum Cosmology, Academic Press, Boston, Mass, USA, 1990.

[37] L. Susskind, "The anthropic landscape of string theory," http://arxiv.org/abs/hep-th/0302219.

[38] P. C. W. Davies, "Multiverse cosmological models," Modern Physics Letters A, vol. 19, no. 10, pp. 727$743,2004$.

[39] M. Tegmark, "Parallel universes," Science and Ultimate Reality: From Quantum to Cosmos, Cambridge University Press, Cambridge, UK, 2003.

[40] D. Deutsch, The Fabric of Reality, Allen Lane, New York, NY, USA, 1997.

[41] H. Everett III, "“Relative state" formulation of quantum mechanics," Reviews of Modern Physics, vol. 29, pp. 454-462, 1957.

[42] I. Montavy and G. Münster, Quantum Fields on a Lattice, Cambridge University Press, Cambridge, UK, 1994.

[43] J. Barata, M. Begalli, and R. Rosenfeld, "Standard model: an introduction," in Particles and Fields, vol. 2000, World Scientific Publishing, Singapore, 1999. 


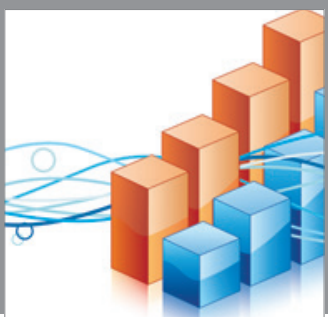

Advances in

Operations Research

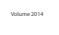

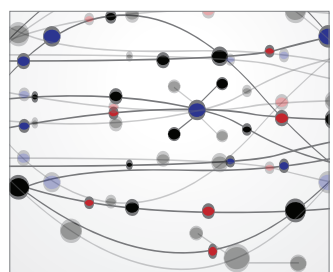

\section{The Scientific} World Journal
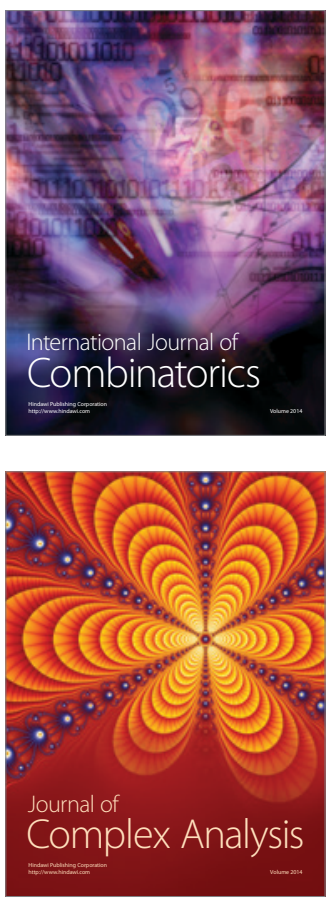

International Journal of

Mathematics and

Mathematical

Sciences
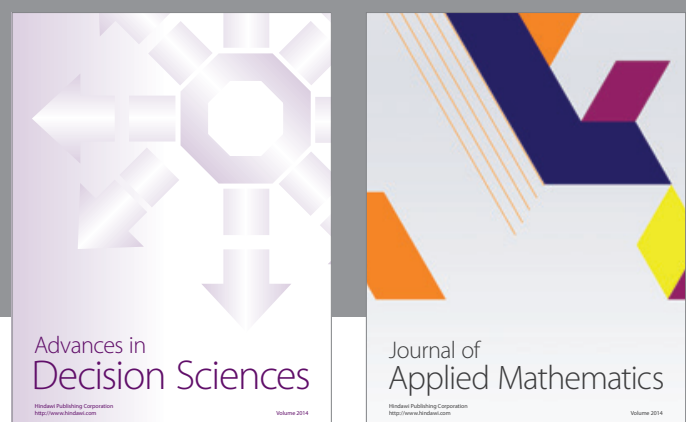

Journal of

Applied Mathematics
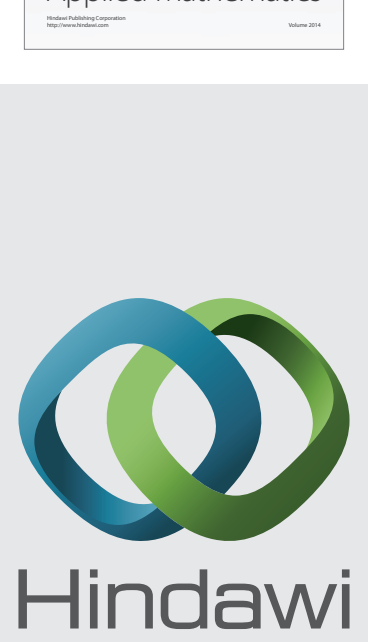

Submit your manuscripts at http://www.hindawi.com
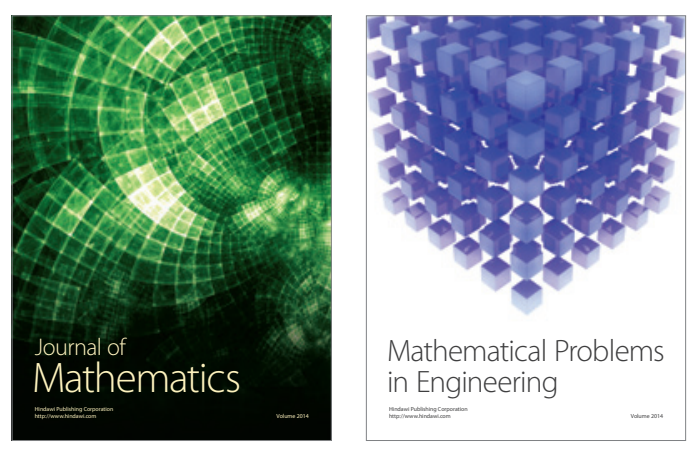

Mathematical Problems in Engineering
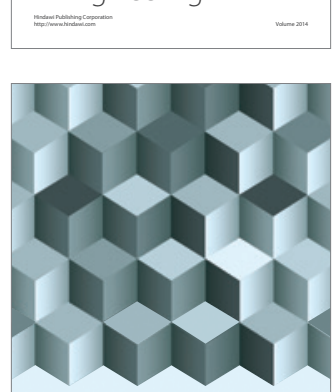

Journal of

Function Spaces
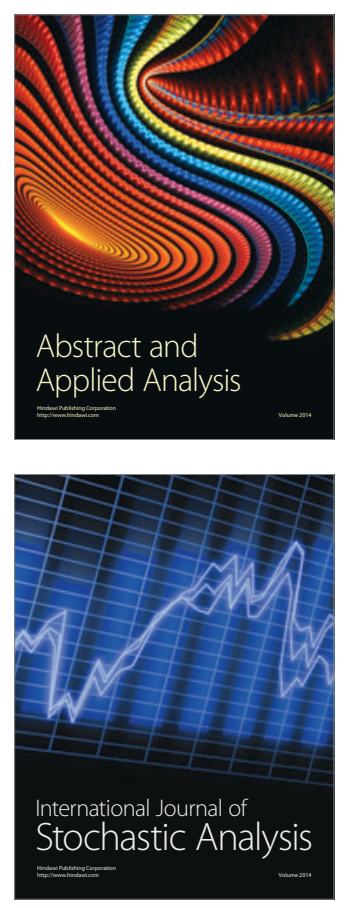

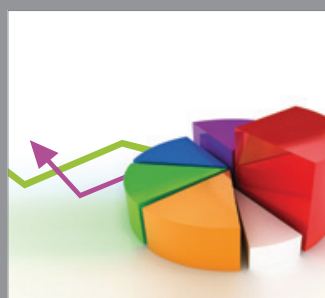

ournal of

Probability and Statistics

Promensencen
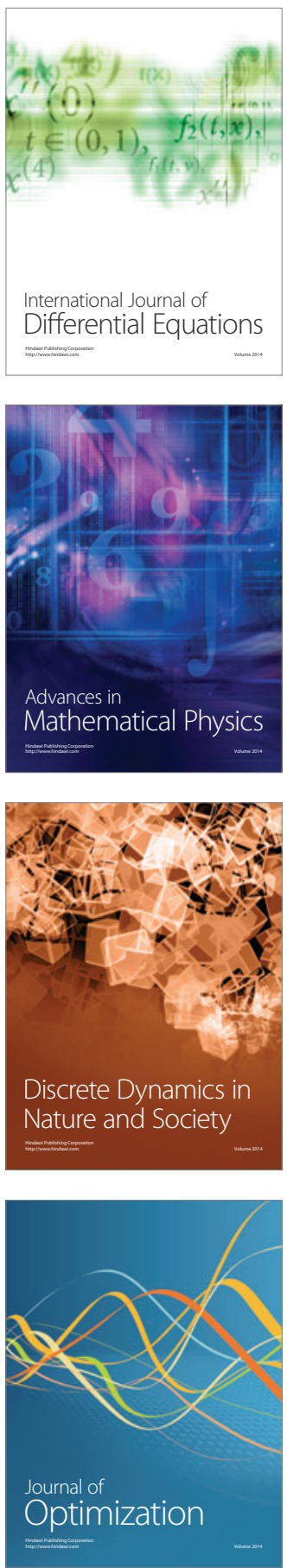